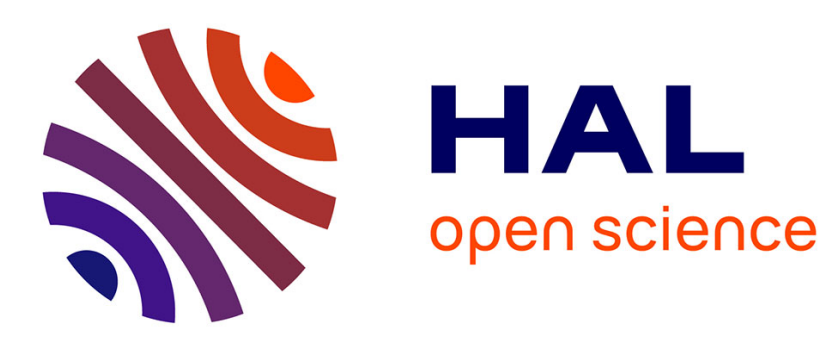

\title{
vol au-dessus d'un tas de cailloux: l'usage en Archéologie de photographies réalisées avec un cerf-volant
}

Olivier Barge, Emmanuelle Régagnon

\section{- To cite this version:}

Olivier Barge, Emmanuelle Régagnon. vol au-dessus d'un tas de cailloux: l'usage en Archéologie de photographies réalisées avec un cerf-volant . Revue Française de Photogrammétrie et de Télédétection, 2017, Spécial Drones, 213-214, pp.95-104. hal-01839261

\section{HAL Id: hal-01839261 \\ https://hal.science/hal-01839261}

Submitted on 14 Jul 2018

HAL is a multi-disciplinary open access archive for the deposit and dissemination of scientific research documents, whether they are published or not. The documents may come from teaching and research institutions in France or abroad, or from public or private research centers.
L'archive ouverte pluridisciplinaire HAL, est destinée au dépôt et à la diffusion de documents scientifiques de niveau recherche, publiés ou non, émanant des établissements d'enseignement et de recherche français ou étrangers, des laboratoires publics ou privés. 


\title{
VOL AU-DESSUS D'UN TAS DE CAILLOUX : L'USAGE EN ARCHÉOLOGIE DE PHOTOGRAPHIES RÉALISÉES AVEC UN CERF-VOLANT
}

\author{
Olivier Barge ${ }^{1}$, Émmanuelle Régagnon ${ }^{2}$ \\ Archéorient, UMR5133, Jalès (07) \\ 1 : olivier.barge@mom.fr \\ 2 : emmanuelle.regagnon@mom.fr
}

\begin{abstract}
Résumé
Les photographies aériennes à échelle très fine et les documents dérivés qu'il est possible d'obtenir présentent, en archéologie, un intérêt évident : les images satellites haute résolution ou les photographies aériennes « traditionnelles" présentent souvent une résolution trop faible pour permettre d'appréhender les objets de recherche. Le recours au cerfvolant permet d'obtenir des images de ces derniers, dans un continuum d'échelles allant du site à l'unité architecturale. Les ortho-photographies et les MNS élaborés à partir des clichés peuvent être intégrés dans des SIG à portée archéologique et ces documents s'avèrent être d'une qualité très satisfaisante en regard des besoins. $A$ la relative rusticité de la méthode répond une facilité et une souplesse de mise en œuvre.
\end{abstract}

Mots-clés : cerf-volant, photogrammétrie, SIG

\begin{abstract}
The air photos at very fine scale are obviously of great interest for archaeology as well as documents which are derived from it: the high resolution satellite imagery and the classical aerial photography have a too low resolution to allow to distinguish the archaeological remains. The use of a kite allows to obtain pictures at various scales, from the architectural detail to the site. Ortho-photos and MNS developed from these images can be integrated in a GIS and their quality is good enough to satisfy archaeologists' needs. The method is rather rustic but the implementation is simple and flexible.
\end{abstract}

Keywords: kite, photogrammetry, GIS

\section{Introduction}

L'imagerie aérienne est une source de données importante en archéologie. En effet, cette dernière est spatiale par nature ; sans localisation, tout vestige ou indice du passé hors contexte perd sa valeur analytique. II est donc naturel que les archéologues aient eu de tout temps recours à la carte et au plan, de même qu'il est naturel que les Systèmes d'Information Géographiques (SIG) soient aujourd'hui très largement utilisés pour l'enregistrement et la structuration des données archéologiques (Rodier (dir), 2012). Les données sont pour la plupart collectées sur le terrain, dont toutes celles, initialement enfouies, qui proviennent des chantiers de fouille. Deux éléments sont en effet incontournables: la description spatiale des vestiges d'une part et la description de leur contexte géographique d'autre part. Permettant d'appréhender en même temps ces deux éléments, l'imagerie aérienne est un support tout à fait pertinent. Dans la plupart des cas, l'image aérienne constitue une donnée, sinon essentielle, du moins complémentaire aux données issues du terrain.

L'archéologie aérienne prit ainsi son essor dés les années 1920 grâce à Antoine Poidebard et ses clichés réalisés au cours de ses survols du Proche Orient, rapidement suivi de précurseurs (Kenneth St Joseph, Raymond Chevallier, ....) dont l'ingéniosité fut d'adapter l'usage du matériel photographique aux contraintes du vol en avion. Les archéologues y trouvèrent alors un grand intérêt et les clichés réalisés à l'époque gardent leur pertinence, les vicissitudes de la modernité ayant souvent davantage dégradé les vestiges que les siècles qui l'ont précédé. Par la suite, des travaux intéressants furent réalisés à partir des photographies issues des missions de couverture aérienne du territoire (Jung, 2006), et plus récemment à partir d'images satellite (Favory, 1998). Ces documents présentent un intérêt évident, mais leur usage est limité à des échelles qui ne sont pas les plus familières aux archéologues. En effet, la plupart des vestiges nécessitent, pour être perçus avec suffisamment de détails, des documents à échelle beaucoup plus fine. Ainsi, l'essentiel des documents aériens utilisés par les archéologues jusqu'à une période très récente furent les photographies obtenues depuis un avion volant à basse altitude. À l'autre bout de la gamme d'échelles, une grue ou un échafaudage ont souvent permis aux archéologues de prendre de la hauteur par rapport à leurs chantiers, mais ces moyens ne permettent que des prises de vues obliques, comme d'ailleurs celles obtenues à partir d'un avion.

Il existait donc un hiatus important dans la gamme des échelles dont les archéologues peuvent faire usage. Par ailleurs, la constitution de SIG se généralisant, les vues zénithales devenaient nécessaires pour être superposées convenablement aux données archéologiques issues des travaux de terrain. A n'en pas douter, l'essor actuel des drones et des moyens d'acquisition de données à distance qui leur sont associés vient opportunément combler ce hiatus.

Cependant, d'autres expériences, certes plus rustiques, leur sont antérieures: les photographies obtenues à 
partir de ballons captifs ou de cerfs-volants. Pour beaucoup, ces expériences furent réalisées au cours de missions archéologiques à l'étranger ${ }^{1}$. C'est le cas de notre propre expérience qui a débuté il y a une douzaine d'années et qui inclut la réalisation de photographies obtenues à partir de ballons captifs et du drone Pixy, probablement obsolète aujourd'hui. Nous voudrions ici relater plus particulièrement celles réalisées à partir de cerfs-volants en insistant sur l'usage qui peut être fait des clichés dans les problématiques archéologiques. A cet égard, l'arrivée récente de logiciels de photogrammétrie simples et ergonomiques a permis d'augmenter significativement l'intérêt des prises de vues. Ni spécialistes d'aérologie, ni photogrammètres, notre préoccupation est de fournir aux archéologues des données pertinentes et correctement structurées; la réflexion procède donc d'un aller-retour permanent entre l'analyse des besoins et l'évaluation des atouts et des contraintes des techniques.

Après avoir évoqué la technique de prise de vues, nous détaillerons les principaux usages qui peuvent être faits des clichés en archéologie avant de proposer une discussion permettant de juger de l'intérêt et des limites des méthodes mises en œuvre. Cette discussion, qui prend en compte les contraintes matérielles, logistiques, administratives ou réglementaires des missions à l'étranger, vaut évidemment pour ce contexte ; Cependant, elle devrait permettre de juger dans quelle mesure et dans quels contextes les cerfsvolants, davantage que concurrents, ne pourraient pas être complémentaires à l'usage des drones dans le cadre de l'archéologie métropolitaine.

\section{La technique}

Après avoir effectué des mesures de lumière et réglé l'appareil photo, on fait décoller le cerf-volant et on lui laisse prendre une trentaine de mètres de hauteur ; il se trouve ainsi au-dessus des turbulences liées à la rugosité du sol. Après avoir jugé de la stabilité du vol, on arrime le dispositif de prise de vue sur la ligne que l'on libère progressivement: on laisse ainsi monter régulièrement l'ensemble (cerf-volant et dispositif de prise de vue) jusqu'à ce que l'appareil photo atteigne l'altitude souhaitée (figure 1). En dehors des éventuelles contraintes réglementaires, il est ainsi possible de positionner l'appareil photo entre une dizaine et plusieurs centaines de mètres au-dessus du sol. On procède alors aux prises de vues. En se déplaçant avec la ligne pendant le vol, l'emprise de la scène change : il est donc possible de multiplier les clichés en décalant successivement l'emprise des photos. Avant la séance de prise de vue, il est utile d'avoir disposé au sol des cibles-repères: une fois leurs coordonnées mesurées, elles permettront le traitement géométrique ultérieur des images. Une fois l'opération de prise de vue terminée, on récupère l'appareil photo, puis le cerf-volant, en passant un mousqueton ou une poulie autour de la ligne dont

\footnotetext{
${ }^{1}$ Un hommage particulier doit ici être rendu à Yves Guichard pour les nombreux sites qu'il a photographié, mais également parce qu'il a fait de nombreux adeptes, dont les auteurs de ces lignes, en divulguant généreusement les principes de sa méthode. Par ailleurs, l'ingéniosité technique de Bernard Noël Chagny a permis de faire progresser la qualité des clichés obtenus : qu'il en soit ici remercié.
}

l'extrémité doit rester fixe et en marchant dans la direction du cerf-volant.

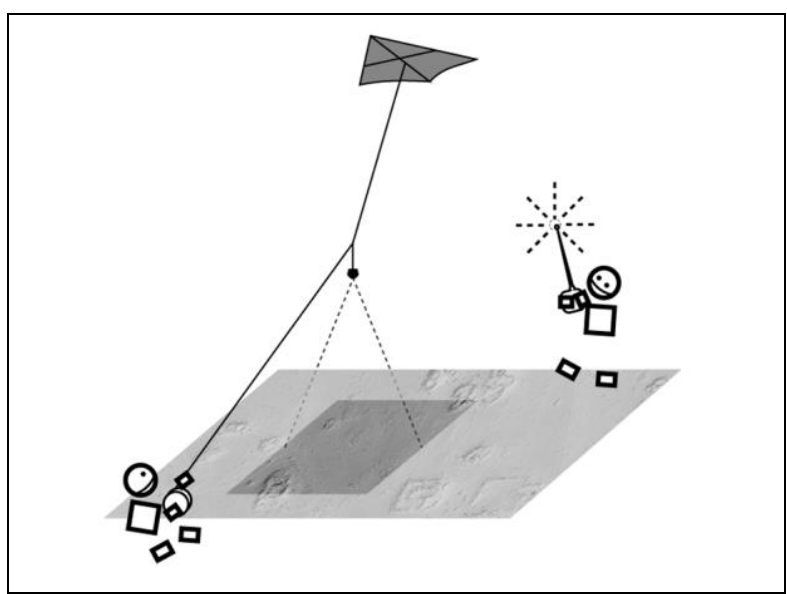

Figure 1 : La technique de vol et de prise de vue

Matériel :

Le matériel se compose du cerf-volant et du dispositif de prise de vue, auxquels on doit ajouter divers ustensiles nécessaires au contrôle du vol: ligne enroulée sur un dévidoir portatif, gants, lunettes de soleil, sangles et mousquetons...

Parmi les modèles de cerf-volant monofil, plusieurs formes conviennent : le delta, le rokaku, ou encore le dopero présentent une stabilité en vol satisfaisante. Leur taille peut varier entre 2 et 4 mètres carrés environ, et elle sera choisie en fonction de la vitesse du vent.

Le dispositif de prise de vue est composé d'un balancier auquel est fixée une nacelle sur laquelle prend place l'appareil photo (figure 2).

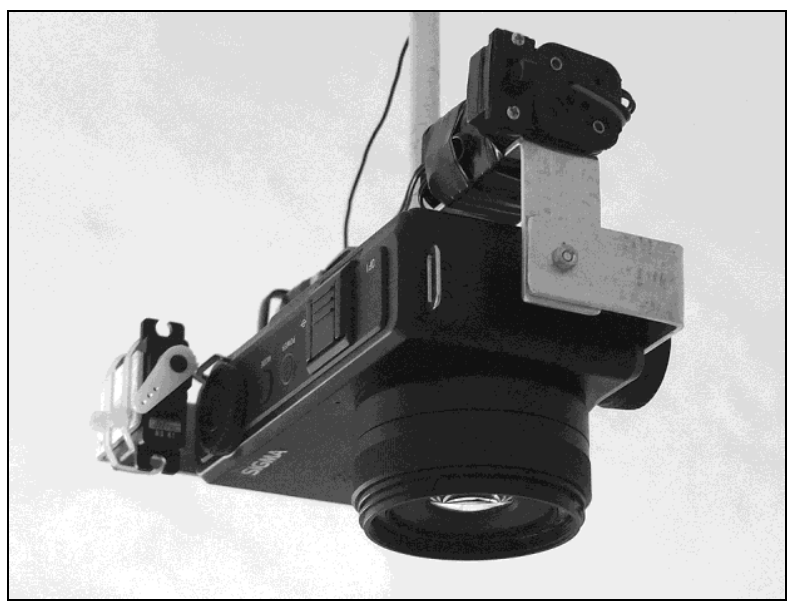

Figure 2: Exemple de nacelle pour des clichés verticaux; on distingue à gauche le servo-moteur qui actionne le déclanchement.

L'ensemble est confectionné à l'aide de profilés aluminium et peut être très simple: la nacelle peut avoir pour unique fonction de maintenir l'appareil photo en visée verticale. En choisissant un déclanchement à l'aide de l'intervallomètre de l'appareil photo, cela est suffisant pour obtenir des vues zénithales. Équiper la nacelle d'une radiocommande pour le déclenchement permet de l'actionner au moment opportun, lorsque l'appareil photo se trouve au-dessus 
de la cible et lorsque la nacelle s'est stabilisée. Les prises de vue obliques nécessitent une nacelle et une radiocommande un peu plus élaborées permettant de régler l'angle par rapport à la verticale et la rotation autour de l'axe du balancier (figure 3). On peut encore y adjoindre une petite caméra de modélisme pour la visée dont le signal est retransmis sur un écran au sol par ondes HF : en pratique peu utile pour les vues zénithales, il s'agit d'un accessoire confortable pour les prises de vues obliques.

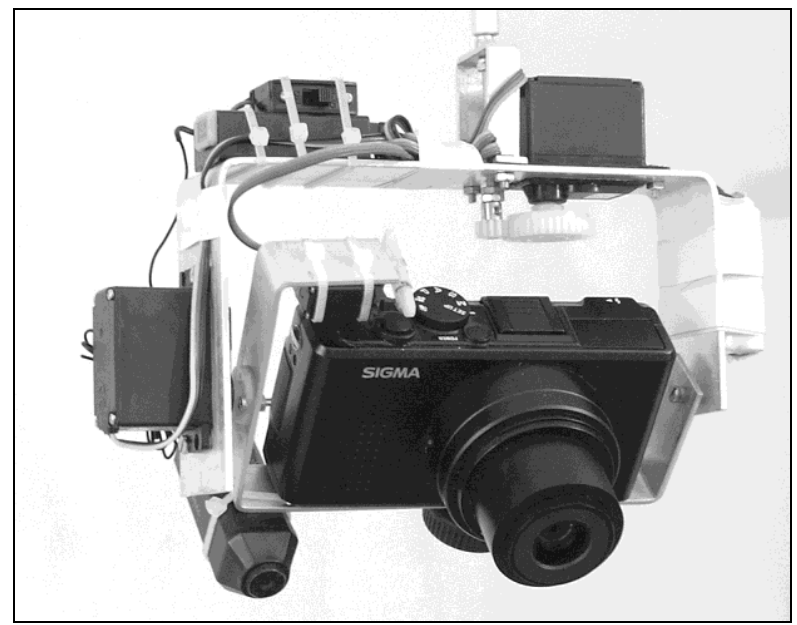

Figure 3 : Une nacelle permettant de réaliser des vues obliques

L'appareil photo, par la qualité de son optique et de son capteur, reste l'élément clé de la réussite de clichés de qualité. II doit combiner un faible poids et la possibilité de débrayer les automatismes de mise au point (point fixe sur l'infini) et d'exposition (mode priorité diaphragme ou priorité vitesse, voire choix d'un couple vitesse/diaphragme). On palie en effet le manque de stabilité relatif de la prise de vue par une vitesse d'obturation élevée (1/800 ème de seconde minimum). Le marché actuel permet d'accéder à des modèles compacts (gamme des compacts experts) qui présentent ces caractéristiques à des prix raisonnables. Les modèles à focale fixe (zoom inutile) sont préférables car ils présentent une meilleure qualité optique et une grande ouverture de diaphragme. La possibilité d'acquérir les images en format brut (raw) permet de corriger les petits écarts de sur ou sous exposition, toujours possibles en l'absence de contrôle direct.

\section{Contraintes :}

La plus évidente des contraintes est liée au vent. Les vitesses exploitables sont approximativement comprises entre 10 et $25 \mathrm{~km} / \mathrm{h}$. Si ces vitesses sont en pratique très fréquentes et que les bulletins météo permettent, dans une certaine mesure, une prévision, il n'en reste pas moins qu'il est hasardeux de planifier de manière stricte un calendrier de prises de vues. Avoir à sa disposition le matériel opérationnel sur le chantier archéologique permet de réaliser d'autres travaux, de relevé par exemple, et de mettre en œuvre les prises de vues lorsque les conditions sont favorables. La technique ne requiert en effet pas de compétences particulière et peut être maitrisée par n'importe quelle personne intervenant sur le chantier: une courte formation et/ou quelques séances d'entrainement préalables suffisent $^{2}$. En outre, cela permet de choisir également la lumière la plus propice: une lumière directe et rasante permet souvent de mettre en valeur les vestiges dans le cas de prises de vue obliques, alors qu'on préfèrera une lumière diffuse (ciels voilés) pour les vues zénithales. La régularité du vent détermine également une mise en œuvre plus ou moins confortable, mais seules de très rares configurations topographiques génératrices de turbulences (ou d'absence de vent à l'altitude souhaitée) l'interdisent véritablement.

La pratique du cerf-volant nécessite un milieu dégagé. Si la présence de quelques arbres ou bosquets épars ne pose pas de problème, il est en revanche impossible de fonctionner en forêt ou en milieu urbain dense. En outre, les lignes électriques constituent une contrainte supplémentaire, moins par le risque qu'elles présentent qu'en limitant les possibilités de déplacement au cours du vol. Ces obstacles limitent de fait les lieux qu'il est possible de survoler sur le territoire métropolitain. En revanche, les zones arides dans lesquelles se déroulent nos travaux archéologiques à l'étranger sont particulièrement adaptées en la quasi absence de ce type de contrainte.

Viennent enfin les contraintes réglementaires. Les cerfs-volants ne sont pas concernés par l'arrêté du 11 avril 2012 relatif aux aéronefs et ils jouissent (encore ?) à ce titre d'une souplesse d'utilisation, au moins pour les altitudes inférieures à $150 \mathrm{~m}$. Dans les pays dans lesquels se déroulent nos recherches, ce genre de règlementation n'existe pas. Par ailleurs, la demande d'une autorisation auprès des autorités n'a été nécessaire qu'à une seule occasion.

Atouts :

Le principal avantage du cerf-volant pour la photographie aérienne réside dans la simplicité du matériel et de la mise en œuvre. Une étude rapide du terrain permet de repérer les possibilités de déplacement au cours du vol afin de couvrir la totalité de l'espace d'étude. On la réalise généralement en même temps que l'on dispose au sol les cibles-repères. Le vol lui-même peut durer quelques minutes ou une à deux heures en fonction de la surface du terrain à couvrir. La simplicité du matériel utilisé limite les risques de panne; un système basé sur l'intervallomètre de l'appareil photo, bien que plus limité et moins confortable, est à ce titre plus fiable qu'une nacelle orientable avec déclenchement et retour vidéo.

L'autre avantage déterminant du cerf-volant est son coût peu élevé. On devra certes acquérir le matériel auprès de divers fournisseurs (cerf-volistes, photographes, modélistes, ...) ou en partie le confectionner, mais la facture totale restera très raisonnable: avec un millier d'euros, on peut rassembler tous les éléments nécessaires, dont un appareil photo de qualité.

Enfin, dans le cas de voyages à l'étranger, le poids et l'encombrement permettent de voyager en avion sans (trop) poser de problèmes de bagages.

La pratique de la photographie aérienne par cerf-volant dépasse largement le périmètre des usages

\footnotetext{
2 Nous dispensons une formation de trois jours inscrite au catalogue de CNRS Formation Entreprises : elle comprend l'apprentissage de la technique de prise de vue et le traitement des clichés.
} 
scientifiques. Les adeptes se constituent en communauté sur internet autour de sites marchands pour le matériel et de forums dans lesquels il est possible de glaner toute une série d'astuces techniques ${ }^{3}$. Les cerfs-volants permettent de réaliser des photographies zénithales et obliques. Les premières sont aptes à un traitement photogrammétrique et autorisent ainsi l'obtention d'ortho-photographies et de modèles numériques de surface. Les secondes, moins abstraites, permettent l'étude patrimoniale des vestiges et présentent souvent des qualités esthétiques permettant leur mise en valeur. Les échelles que l'on peut aborder, fonction de l'emprise des clichés et donc de la hauteur du cerfvolant, sont diverses: depuis celle du chantier de quelques mètres carrés, à celle d'un site de plusieurs dizaines d'hectares. La nature et la qualité des documents produits ouvrent donc, a priori, à de nombreux usages dans le cadre de la recherche en archéologie.

\section{Les usages}

Afin de rendre compte de la diversité des usages possibles des clichés aériens et des documents que leur traitement permet d'obtenir, nous présenterons quelques exemples issus de plusieurs cas d'étude. A cette occasion, on pourra juger de l'exactitude des documents en regard des objectifs de recherche, et/ou de leur pertinence.

\section{Illustrations pour la description patrimoniale des vestiges:}

Nous n'insisterons pas sur cet usage bien connu des photographies aériennes, qui n'est du reste pas propre à l'archéologie. Cependant, pour classique qu'il soit, l'usage de photographies aériennes obliques permet une description des vestiges dans leur organisation spatiale puisque, vus du haut, ils se révèlent sous une perspective inaccessible pour un observateur au sol. Dans le même temps, les détails de l'architecture, les matériaux employés, la composition avec le relief, etc. sont perceptibles comme au sol. Ces documents permettent donc d'appréhender les vestiges dans leur spatialité sans pour autant devoir faire la démarche intellectuelle et cognitive du passage au plan.

Cette vision particulière apporte une aide réelle à l'archéologue pour la compréhension et l'interprétation d'un site. Par ailleurs, moins abstraites que le plan, les photographies aériennes obliques sont très prisées du grand public et présentent donc des vertus pédagogiques dans les démarches de communication que le chercheur doit entreprendre.

Le cerf-volant permet de réaliser ce genre de cliché rapidement et à moindre coût.

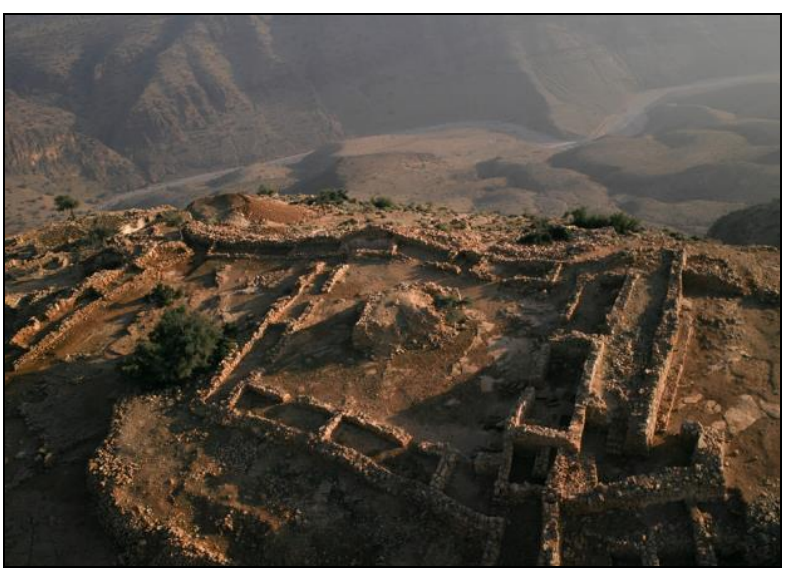

Figure 4 : Vue oblique de la zone de commandement de la citadelle d'Igîlîz (XII ème $\mathrm{S}$., Anti Atlas, Maroc).

\section{La description du contexte topographique d'un site :}

Le contexte topographique d'un site, l'insertion des vestiges dans le relief, est un élément incontournable pour comprendre ses fonctions, les relations avec le territoire, ou encore les partis pris architecturaux des constructeurs du passé. Traditionnellement réalisé à l'aide d'une station totale qui mesure les coordonnées ( $\mathrm{x}$, y et $\mathrm{z}$ ), la description topographique («le plan topo ») est d'autant plus longue et fastidieuse que la résolution souhaitée est grande. Pour des sites étendus, cela peut représenter des mois de travail pour deux personnes sur le terrain. L'usage récent de GPS différentiels permet d'atteindre des précisions équivalentes en réduisant le temps de saisie. II s'avère que le traitement photogrammétrique des photos réalisées au cerf-volant permet d'obtenir des documents avec une résolution incomparablement plus fine alors que le temps pour les réaliser correspond à une séance de prise de vue et quelques heures de traitements.

La première de nos réalisations de MNS a concerné la nécropole d'Adam en Oman (Giraud, 2012). Au pied et au sommet d'une série de buttes témoins, 133 tombes furent construites à différentes périodes sur une durée d'une vingtaine de siècles. II s'agissait de relever ces tombes et de les rattacher à une période quand cela était possible. Le contexte topographique avait une importance particulière, puisqu'on observe des préférences d'implantation entre les périodes. Avant de traiter les données au bureau, nous nous étions assurés de repartir du terrain avec les données nécessaires et avions enregistré des entités altimétriques à l'aide d'un GPS différentiel : les deux méthodes - production d'un modèle TIN à partir des entités GPS, rastérisation et filtrage d'une part, traitement photogrammétrique des photos cerf-volant d'autre part - ont donc été mises en œuvre conjointement. La comparaison des ombrages (hillshade) dérivés des MNT, qui permet de mettre en lumière la sensibilité (Cauvin et al., 2007 a), ne laisse aucun doute : la description de la topographie se révèle beaucoup plus fine sur le MNS photogrammétrique que sur le MNT GPS (figure 5).

\footnotetext{
${ }^{3}$ Sites et forums accessible aisément à partir d'une recherche sur « KAP » : Kite Aerial Photography
} 


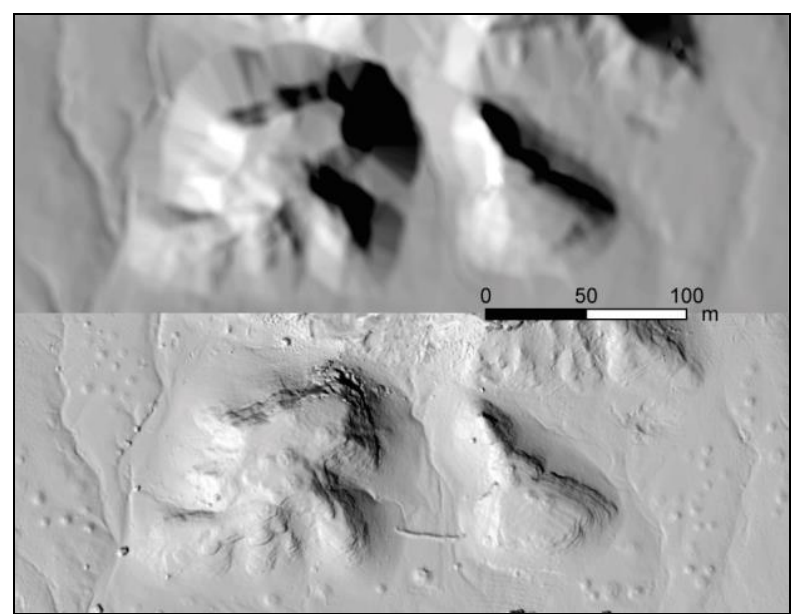

Figure 5 : La sensibilité comparée des deux méthodes (en haut, MNT obtenu à partir de mesures DGPS, en bas, MNS photogrammétrique) révélée par les ombrages (hillshade).

Bien davantage de détails sont perceptibles. Dans le cadre de la recherche concernée, tous ces détails n'étaient pas nécessaires puisque la représentation la plus pertinente pour contextualiser les tombes passe par la production de courbes de niveau qu'il a fallu généraliser pour davantage de lisibilité cartographique. On peut donc conclure que le MNS produit à partir des photos cerf-volant est non seulement suffisant, mais presque trop détaillé en regard des besoins de la recherche (figure 6). De quoi regretter les quatre jours à arpenter la montagne avec le GPS ! repérer aisément et de pouvoir, quasiment au pixel près, en déterminer le centre par ailleurs mesuré au GPS différentiel sur le terrain. Une partie de ces points a été utilisée comme points de calage pour réaliser des modèles photogrammétriques à partir de 63 photos, l'autre comme points de contrôle. Le logiciel Agisoft Photoscan a été utilisé pour la production de différents modèles desquels ont été générés des MNS et des ortho-photos. Nous avons fait varier le nombre de points de calage ainsi que la méthode (smooth ou sharp) et la résolution des modèles $3 \mathrm{D}$, puis avons mesuré l'écart entre les points de contrôle et leur position sur l'ortho-photo (x; y) et le MNS (z). La précision $(68 \%)$ obtenue pour l'ensemble de ces modèles est en moyenne de $6.36 \mathrm{~cm}$ (figure 7).

\begin{tabular}{|l|l|l|}
\hline \multirow{2}{*}{$\begin{array}{l}\text { 11 points de calage } \\
\text { sharp }\end{array}$} & $\mathrm{x}, \mathrm{y}$ & $5.9 \mathrm{~cm}$ \\
\cline { 2 - 3 } & $\mathrm{z}$ & $7.8 \mathrm{~cm}$ \\
\hline \multirow{2}{*}{$\begin{array}{l}11 \text { points de calage } \\
\text { smooth }\end{array}$} & $\mathrm{x}, \mathrm{y}$ & $5.0 \mathrm{~cm}$ \\
\cline { 2 - 3 } $\begin{array}{l}23 \text { points de calage } \\
\text { sharp }\end{array}$ & $\mathrm{z}, \mathrm{y}$ & $7.9 \mathrm{~cm}$ \\
\cline { 2 - 3 } $\begin{array}{l}\text { 23 points de calage } \\
\text { smooth }\end{array}$ & $\mathrm{z}$ & $7.0 \mathrm{~cm}$ \\
\cline { 2 - 3 } & $\mathrm{x}, \mathrm{y}$ & $7.8 \mathrm{~cm}$ \\
\hline
\end{tabular}

Figure 7 : Exactitudes évaluées de l'ortho-photo $(x ; y)$ et du MNS (z) pour une précision de $68 \% ; 63$ photos à environ 100 mètres de hauteur ont été utilisées.

Elle varie faiblement en fonction des paramètres utilisés, le nombre de points de calage étant celui qui semble avoir le plus d'incidence sur les résultats: augmenter le nombre de points de calage améliore la

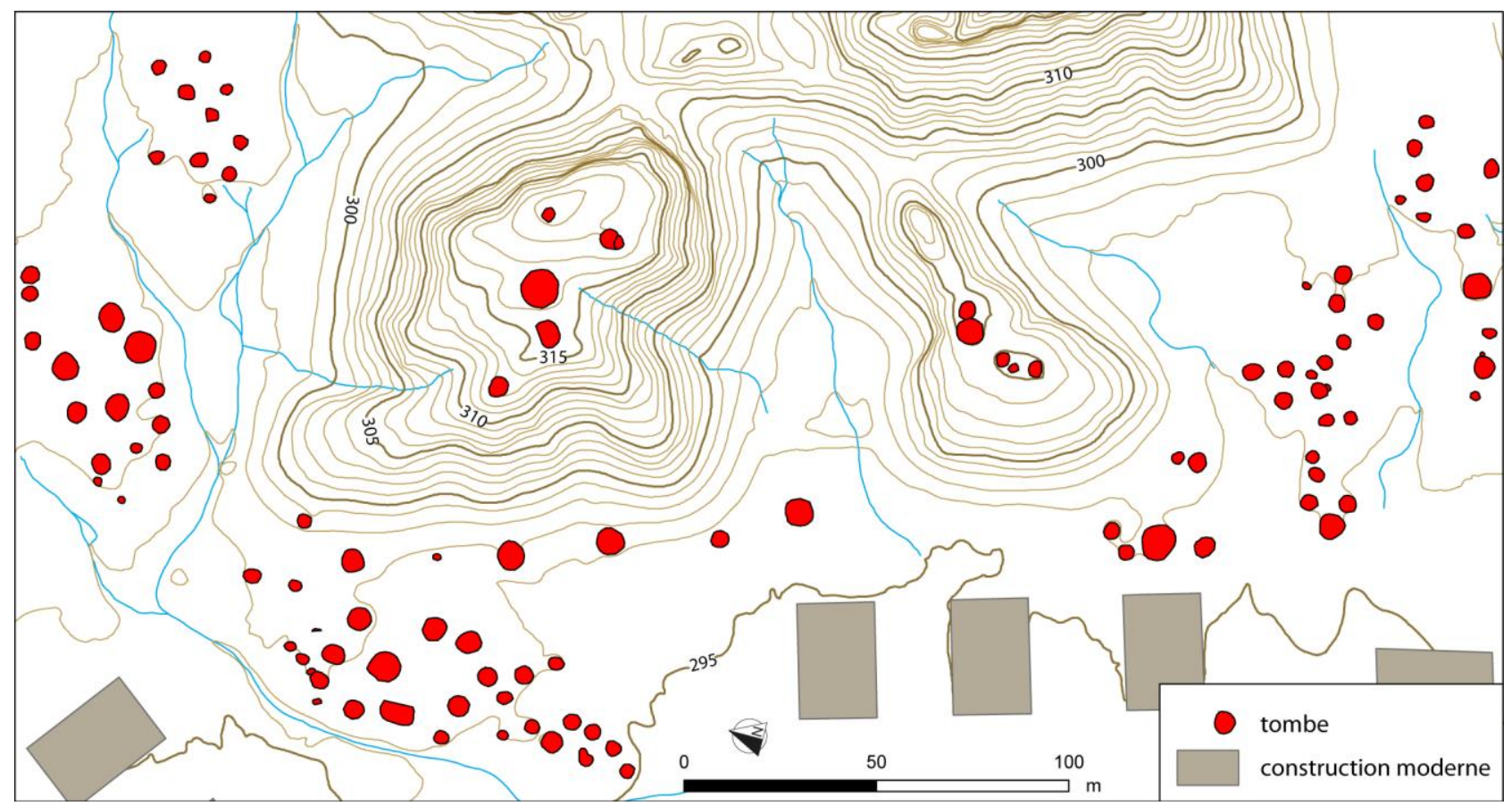

Figure 6 : Les courbes de niveau généralisées (équidistance : $1 \mathrm{~m}$ ) pour la description topographique de la nécropole d'Adam (Partie sud)

La sensibilité est une chose, la précision en est une autre. Avant la séance de prise de vue, nous avions disposé au sol 120 assiettes de pique-nique qui constituent des cibles repères, à cette échelle, parfaitement adaptées. Elle représentent une dizaine de pixels sur les images permettant à la fois de les précision en z. En outre, la précision obtenue lors de ces tests est très voisine de la RMS estimée par le logiciel.

Sans détailler ce test, il montre que la précision que l'on peut attendre de la méthode est très largement suffisante en regard du besoin, surtout si on la rapporte 
à la surface concernée: elle permettrait une cartographie des 6 ha au $1 / 300$ alors que, pour apprécier l'implantation topographique des tombes, les cartes produites le sont à beaucoup plus petite échelle (figure 6).

On peut donc conclure que la méthode de production de MNS à partir de photos cerf-volant est très satisfaisante pour la description topographique des sites. Elle offre un rendu beaucoup plus détaillé que ceux obtenus par les méthodes traditionnelles, avec un gain de temps impressionnant. Sur le site d'Igîlîz, au Maroc, 256 photos ont permis d'obtenir un MNS de près de 30 ha avec une RMS estimée par le logiciel à 4 $\mathrm{cm}$, alors qu'antérieurement, un levé à la station totale de 30 jours sur le terrain avait permis de générer des courbes de niveau sur moins de 10 ha.

\section{L'ortho-photo et le MNS dans le SIG:}

Le traitement photogrammétrique des photos réalisées au cerf-volant permet d'obtenir une ortho-photographie qui peut directement être importée dans le SIG. Dans la mesure où les coordonnées des points de calage ont été mesurées dans un système de coordonnées renseigné de ses paramètres ${ }^{4}$, l'ortho-photo se trouve automatiquement géoréférencée, et est donc superposable à toute autre donnée géoréférencée.

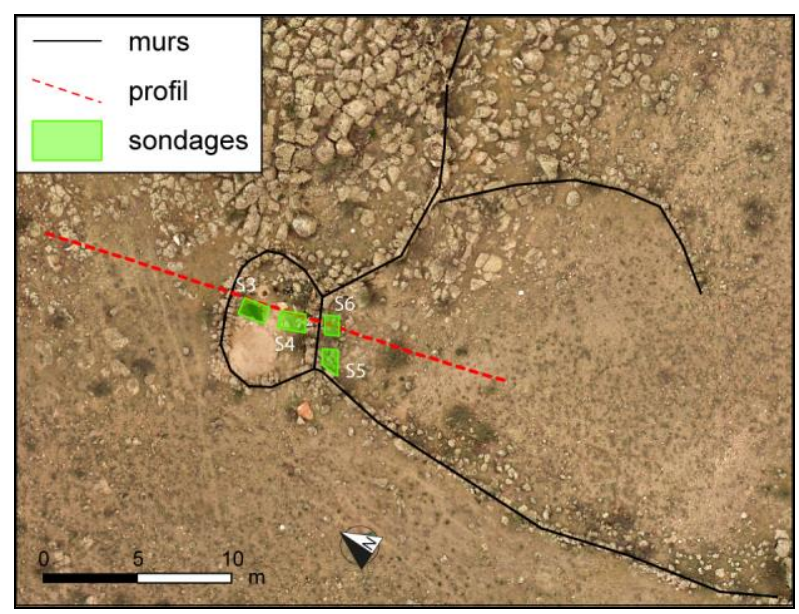

Figure 8 : Superposition d'entités vecteur et de l'orthophoto ; Ici, les travaux effectués (profil et sondages) dans le cadre de l'étude de structures cynégétiques anciennes (les « desert kites »).

Avant l'arrivée des logiciels récents de photogrammétrie, il était possible de géoréférencer les photos en utilisant des points de calage dans l'interface du SIG. Cette méthode, non fondée sur la prise en compte du relief, était évidemment moins fiable. Des comparaisons avec l'ortho-photo ont montré qu'elle était toutefois parfaitement acceptable en utilisant une méthode de déformation élastique et à condition que le rapport dénivelé/emprise ne soit pas trop grand, ce qui ne permettait pas cet usage aux plus grandes échelles, surtout lorsque les vestiges comportaient des élévations importantes. La mise en œuvre était cependant fastidieuse puisque la validité du résultat

\footnotetext{
${ }^{4}$ A l'étranger, le système de coordonnée utilisé est celui en vigueur dans le pays d'accueil. Cependant, les cas où ce dernier n'existe pas ou dont les paramètres sont tenus secrets sont fréquents. Nous utilisons alors le système UTM.
}

était conditionnée par un grand nombre de points de calage. En outre, l'opération de mosaïquage qui s'avérait parfois nécessaire impliquait de l'effectuer en dehors de l'environnement SIG et passait souvent par des manipulations manuelles. Cette dernière opération, effectuée dans le logiciel de photogrammétrie en même temps que les clichés sont corrigés de leurs déformations géométriques, s'avère parfaite.

Ainsi, l'ortho-photographie devient une couche d'information dans le système, superposable aux données archéologiques acquises par ailleurs sur le terrain. Ces dernières, en mode vecteur, délimitent et décrivent les vestiges (emprise, matériaux, appareillage et hauteur d'un mur, emprise et nature d'une unité stratigraphique par exemples). En phase exploratoire des données, l'archéologue dispose donc conjointement des données formalisées, mais également de leur image zénithale en même temps que de leur contexte géographique (figure 8).

Si l'ortho-photo n'est pas suffisante pour décrire les vestiges eux-mêmes (voir infra), elle peut parfois l'être pour la description du contexte: limites de constructions modernes, emprise des travaux, position d'un fossé, d'un affleurement, d'un axe de drainage, d'un cours d'eau, etc. En fonction de leur plus ou moins grande importance en regard des questions archéologiques posées, ces éléments de contexte peuvent être vectorisés en appui sur l'ortho-photo, évitant une saisie bien plus longue sur le terrain

Davantage que cette possibilité de délimiter des unités à partir de l'ortho-photo, c'est le croisement de la lecture de cette dernière avec les autres informations, rendu possible par la superposition exacte, qui lui donne sa valeur dans le système d'information. L'analyse de l'image des éléments architecturaux, par exemple, pourra être confrontée à leur description sur le terrain. En cliquant dans l'image, on accède aux informations attributaires des entités archéologiques qui lui sont superposées. De la même manière, un clic en tout point de l'image permet d'en connaitre l'altitude, cette dernière information provenant de la superposition du MNS.

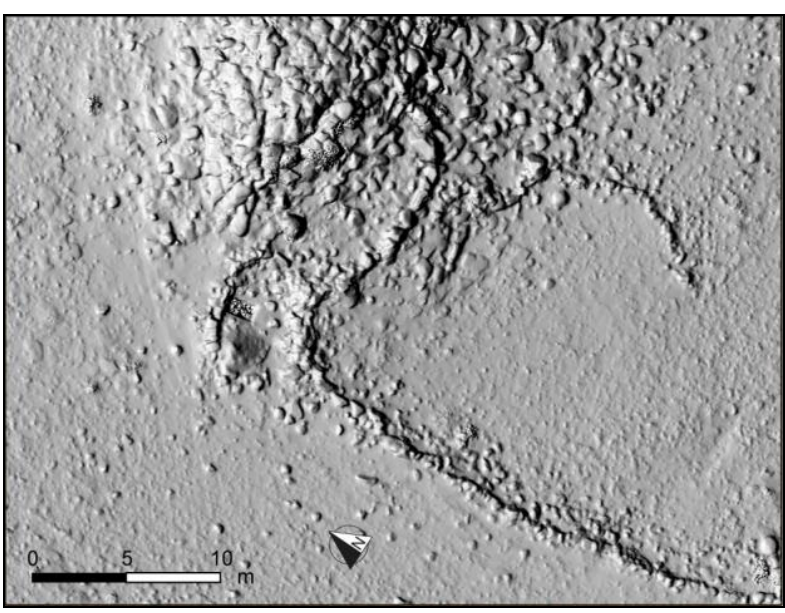

Figure 9: L'insertion des « desert kites» dans le paysage est un élément important pour la compréhension de son fonctionnement; l'ombrage permet de distinguer la facture de la construction dans le contexte d'affleurements basaltiques. 
Cette manière d'explorer les données permet une analyse fine des vestiges et les données provenant du cerf-volant en donnent une image plus riche. Les temps de saisie sur le terrain étant important, on est souvent conduit à "généraliser» les vecteurs qui décrivent les vestiges. Par exemple, un mur pourra être décrit par une ligne à laquelle sera associée sa hauteur et sa largeur. Afficher l'ortho-photo permet d'en discerner toutes les pierres qui le constituent et ses irrégularités (figure 8). Afficher l'ombrage du MNS en donne une autre image, parfois très instructive, comme c'est le cas de l'étude des structures en pierre sèche (figure 9).

D'autres éléments, qui ne peuvent ou ne doivent pas nécessairement être explicitement décrits dans le système d'information, peuvent avoir leur importance et être analysés à partir de la photo. II en est ainsi des changements plus ou moins progressifs de couleur, de texture ou de granulométrie du sol, comme des variations de densité ou de nature de la végétation. Ces changements et ces variations peuvent être des indicateurs de vestiges enfouis ou encore de travaux passés de nivellement ou de drainage. L'analyse de ces indices procède d'une méthodologie par ailleurs décrite, utilisée et validée depuis longtemps (Delétang, 2006).

Les informations et les documents issus des images acquises à l'aide du cerf-volant permettent, parfois en proportion importante, d'alimenter les rapports et les publications de l'archéologue : cartes (courbes de niveau et éléments de contexte), ortho-photos, documents dérivés du MNS (ombrages par exemple) et photos obliques constituent des illustrations pertinentes.

\section{Discussion}

\section{La précision en question}

Nous avons détaillé les précisions obtenues lors d'un test correspondant à l'une de nos toutes premières réalisations. Un plus grand nombre de photos permet assurément d'obtenir de meilleures précisions comme probablement l'usage de logiciels plus sophistiqués. Toutefois, comme nous l'avons souligné, les précisions obtenues restent plus que suffisantes en regard des objectifs attendus. En effet, les précisions n'ont de sens qu'en fonction de l'échelle abordée et surtout des besoins. II n'existe pas de précision bonne en soit, seulement des précisions adaptées ou non aux objectifs qu'on se fixe. II serait plus pertinent d'employer le terme d'exactitude, écart qui existe entre une position réelle et celle que l'on enregistre (Cauvin et al., 2007 b) : en effet, cela permettrait, pour une échelle donnée, de définir à l'avance l'exactitude acceptable pour un besoin donné. Dans le cas de la nécropole d'Adam (6 ha) que nous avons détaillé, une exactitude de l'ordre du décimètre était très largement suffisante: les précisions obtenues (68\%) correspondent à une exactitude inférieure au décimètre.

La production d'ortho-photographie est sans conteste plus satisfaisante que le géoréférencement, tout en étant moins fastidieuse. Elle permet, dans la même démarche, l'obtention de MNS dont l'intérêt est au moins aussi important. L'usage de la photogrammétrie moderne a donc augmenté radicalement la valeur ajoutée des photos obtenues à l'aide de cerf-volant. L'usage d'autres moyens d'acquisition permettrait sans doute une meilleure régularité des prises de vues et partant, une meilleure précision. De même, photoscan n'est sans doute pas l'outil le plus performant, mais il permet d'être facilement pris en main par un nonspécialiste et fournit des interfaces d'exportation vers les formats de données exploitées en SIG tout à fait confortables. Tous les sites archéologiques qui font l'objet de recherche ne sont pas prestigieux ou ne présentent pas un caractère patrimonial propre à devoir enregistrer des données à la plus haute précision possible. Si on conçoit qu'un site comme la grotte Chauvet, pour des raisons patrimoniale comme de conservation, nécessite la mise en œuvre des techniques les plus affutées, l'écrasante majorité des sites étudiés - et des questions qu'ils posent - se satisfont amplement de techniques plus rustiques.

Faire progresser les précisions obtenues ne constitue pas, dans le cadre de notre pratique, une priorité ; nos problématiques archéologiques n'en requièrent tout simplement pas davantage. En revanche, le dialogue avec les archéologues - montrer quels sont les potentiels de la méthode d'une part, mieux comprendre en quoi les documents produits peuvent faire progresser les questions qu'ils se posent d'autre part nous semble une voie de recherche méthodologique prometteuse.

Le mythe du "terrain au bureau » :

Les résultats obtenus montrent que les informations susceptibles d'être dérivées des photos réalisées à l'aide d'un cerf-volant présentent une bonne qualité et une grande pertinence en regard des objectifs de recherche. Toutefois, surestimer l'usage que l'on peut faire de ces informations constitue un risque qui n'est pas propre à la méthode, ni probablement à l'archéologie. La précision et la finesse des informations leur confèrent un réalisme surprenant, et il serait tentant de les considérer comme suffisantes. Comme pour d'autres moyens d'acquisition à distance (scan laser $3 \mathrm{D}, \ldots$ ), l'erreur consisterait à penser que la qualité et la quantité des données qu'apportent ces méthodes dispensent d'investigations sur le terrain. En archéologie, et sans doute dans d'autres disciplines, une donnée ne devient information qu'à partir du moment où la réalité qu'elle représente est interprétée et formalisée. Les réalités physiques auxquelles on s'intéresse, mesurées avec grande précision par ces méthodes, peuvent, dans une certaine mesure, être interprétées à partir des données produites, et c'est bien la raison pour laquelle ces méthodes nouvelles apparaissent si précieuses. Mais cette interprétation/formalisation ne peut être que partielle.

En archéologie, l'acquisition à distance ne peut concerner que les vestiges perceptibles en surface. L'autre partie des données est enfouie et c'est cette partie, presqu'exclusivement, qui ouvre à la chronologie. Mais même en ne considérant que l'information de surface, cette dernière n'est pleinement accessible que par une investigation de terrain. Les vestiges y sont appréhendés par une infinité d'indices dimensions, nature, calibre, texture, agencement des matériaux, relation éventuelle avec du mobilier, relation avec l'environnement, ... - qui ne sont perceptibles conjointement qu'in situ. La manière par laquelle une réalité archéologique est identifiée et caractérisée est difficilement formalisable, elle relève de ce que l'on peut appeler, à défaut de définition précise, «l'œil de l'archéologue ». Mais cette expertise archéologique qui 
transforme la donnée en information, ne peut se réaliser seulement à travers une représentation des réalités physiques, aussi détaillées et précises qu'elles puissent être, le terrain restant nécessaire. Pour illustrer ce propos général, nous donnerons un exemple concernant l'usage des ortho-photos.

A partir d'une ortho-photo réalisée sur un des secteurs d'Igîlîz (résolution $0.025 \mathrm{~m}$ ), on pourrait penser pouvoir restituer le plan de maisons disséminées sur le versant méridional du site. De ces vestiges, il ne reste que partiellement les arases de murs, exceptionnellement une élévation. Ces derniers ont été mis en lumière par un nettoyage de surface (ablation de la végétation, dégagement des arêtes de murs). Sur l'ortho-photo, on perçoit très bien ces vestiges. Pour autant, la vectorisation par superposition de l'ortho-photo ne donnerait qu'un plan très incomplet. Certains murs ne sont pas perceptibles : soit les pierres qui le constituent ne sont pas clairement visibles (confusion avec la couleur du sol, absence d'ombres), soit les indices qui permettent sur le terrain de le reconstituer n'apparaissent pas : alignement des arêtes des pierres qui forment un parement, position d'un angle, ou encore nivellements. A l'inverse, les alignements apparents de certaines pierres pourrait conduire à les interpréter comme une construction alors qu'ils sont en réalité fortuits, en particulier à cause de la présence fréquente de pierres qui ne sont pas en place (ce que l'on juge facilement sur le terrain). Encore le cas évoqué est-il plutôt favorable par le fait qu'un nettoyage de surface a été réalisé (figure 10). Par ailleurs, un relevé de terrain permet l'enregistrement conjoint de données associées: numéro des bâtiments, présence d'une particularité, présence d'artefacts en surface, ... ce que ne permettent pas les méthodes d'acquisition à distance.

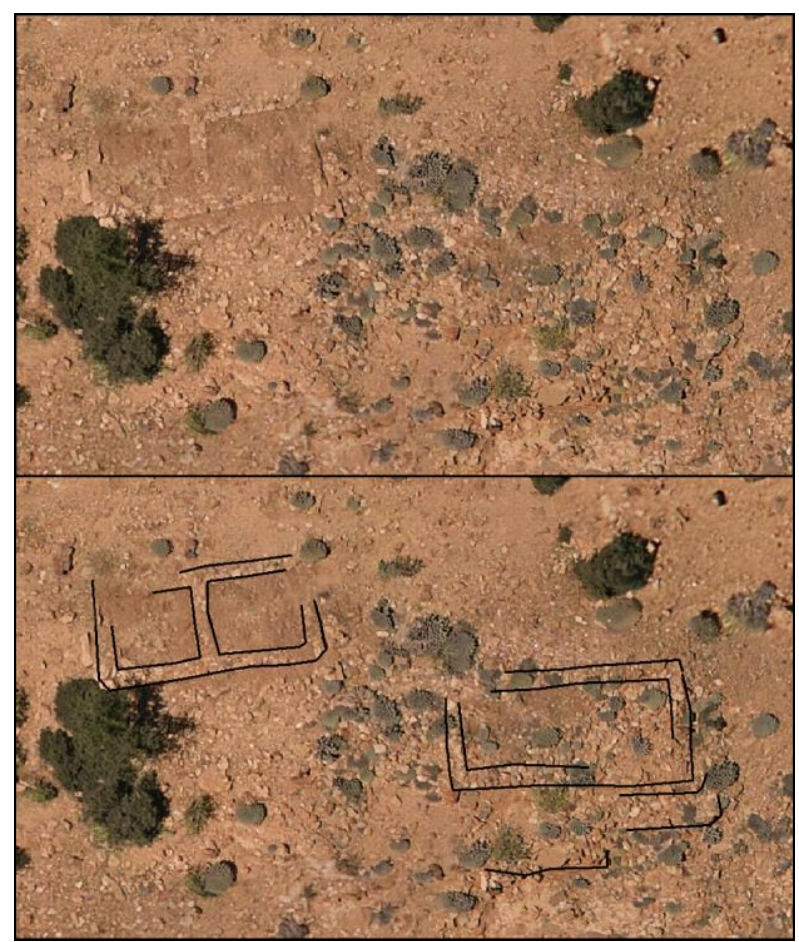

Figure 10 : Ortho-photo de bâtiments dont il ne reste que l'arase des murs (en haut); superposition du relevé réalisé sur le terrain (en bas).
Cet exemple montre qu'aucune technique d'enregistrement à distance, aussi précise soit-elle, ne remplace l'enregistrement sur le terrain. En revanche, comme nous l'avons mentionné plus haut, les données issues du cerf-volant offrent des images complémentaires et peuvent améliorer l'analyse des vestiges. Elles peuvent aussi parfois apporter d'autres indices précieux.

Des indices précieux et parfois inattendus :

Tout d'abord, l'ortho-photographie peut révéler des erreurs ou des omissions dans les relevés. II peut arriver, pour diverses raisons dont celles liées aux conditions souvent rustiques du terrain, qu'une erreur se glisse dans le plan des vestiges. L'orthophotographie devient alors un révélateur sans concession. Les relevés sont réalisés de manière cumulative, souvent en fonction de l'avancée de la mise au jour des vestiges, parfois au cours de plusieurs campagnes successives ou par plusieurs personnes différentes. II nous est arrivé à plusieurs reprises de constater, sur les plans, des décalages de certains murs ou certains bâtiments, bien que la topologie des éléments reste correcte. Ces décalages sont difficilement perceptibles sur le terrain et peuvent conduire à des incohérences inexpliquées. L'orthophotographie permet alors d'en prendre conscience et de les corriger.

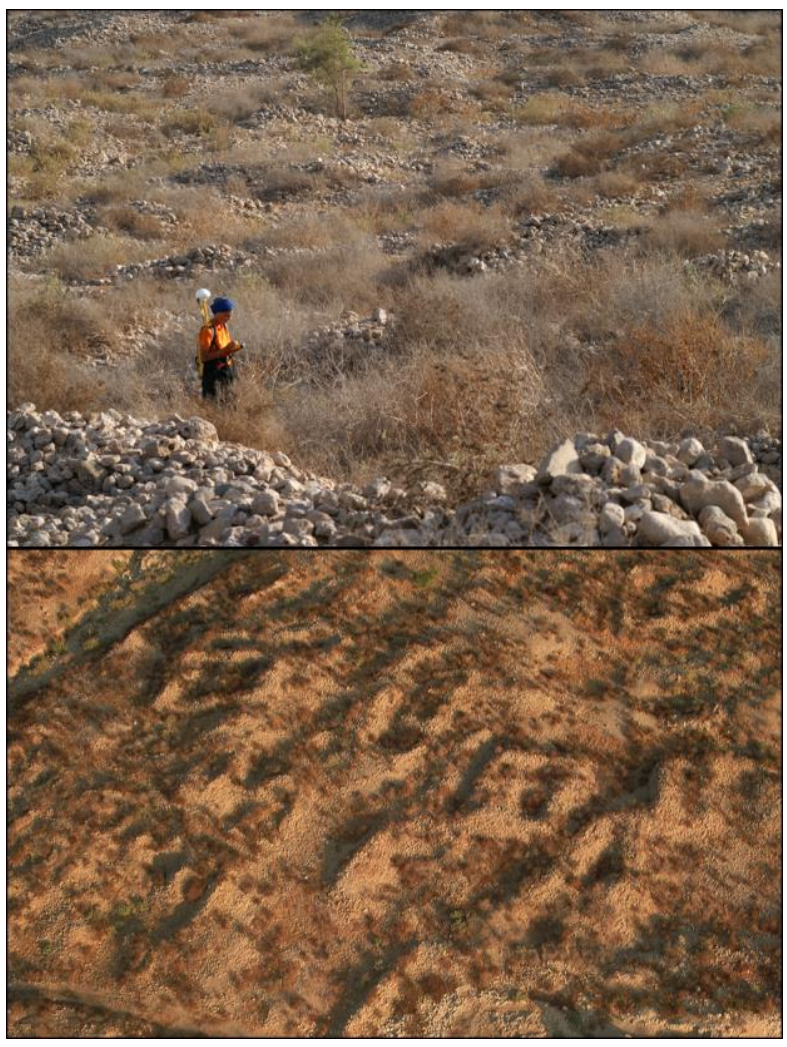

Figure 11 : Les ruines de la ville médiévale de Qalhat (Oman) vues au sol (en haute) et à partir d'un photo cerf-volant (en bas).

II est des cas où les vestiges sont constitués de ruines et ou l'enchevêtrement des divers écroulements rend la reconstitution du plan très difficile, voire impossible. La perception au sol ne permet pas de retrouver la cohérence des indices qui subsistent sous la forme d'alignements d'accumulation ou de segments de murs 
encore en place. L'ortho-photographie peut alors s'avérer être d'un grand secours. Tel fut le cas de la ville médiévale de Qalhat (Oman) ou l'organisation des bâtiments, ou tout au moins d'ensembles de bâtiments, se perçoit très bien vue du ciel, alors que la perception au sol ne révèle qu'un chaos inextricable (figure 11).

Nous avons alors procédé en deux temps: nous avons d'abord réalisé un dessin provisoire des bâtiments que l'on voyait ou devinait sur l'ortho-photo, tout indice cohérent étant pris en compte. Assistés de ce protoplan (géoréférencé), transféré sur le carnet de terrain qui contrôle le GPS d'une part, imprimé d'autre part, nous sommes retournés sur le terrain : nous avons pu contrôler les indices perçus sur l'ortho-photo, sélectionner ceux qui se révélèrent pertinents et, forts d'une nouvelle cohérence, découvrir et enregistrer d'autres indices pour reconstituer les bâtiments. Le plan produit a posteriori par reconstitution de pièces du puzzle fut associé à un critère calculé de fiabilité en fonction des indices utilisés (Barge et Régagnon, 2011).

D'autres indices, parfois fortuits, peuvent être perçus sur l'ortho-photo (voire sur les photos obliques) et/ou sur le MNS. II est impossible de dresser une liste ou une typologie des indices que la chance peut permettre d'obtenir, et s'il ne faut pas s'attendre à découvrir des pyramides, certains indices peuvent conduire à des découvertes inattendues. Par exemple, sur l'orthophoto générale de la montagne d'Igîlîz, on distingue des traces linéaires sombres formant des lignes perpendiculaires. II s'agit de l'image d'une végétation et d'un sol différent de l'environnement, indiquant peut être la présence d'un bâtiment ayant été volontairement démantelé. Toujours à Igîîzz, on perçoit dans le paysage des nivellements s'appuyant sur la topographie naturelle et permettant les accès et les circulations à l'intérieur des fortifications. Sur le terrain, ces nivellements sont peu perceptibles car le doute d'une confusion avec une forme naturelle subsiste toujours. Les ombrages produits du MNS les révèlent beaucoup plus clairement (figure 12).

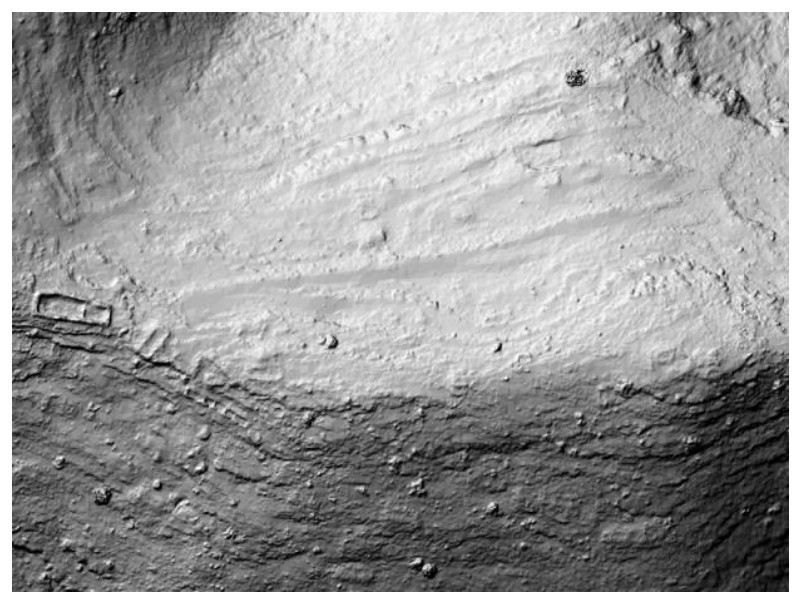

Figure 12: Au centre de l'image (ombrage du MNS), on perçoit nettement le nivellement correspondant à une rampe d'accès: la forme linéaire, de largeur constante, recoupe partiellement les affleurements naturels.
La souplesse de la méthode

A la sophistication des techniques, à la quantité et/ou la qualité des données qu'elles produisent, vont souvent de paire un coût et des contraintes de mise en œuvre en proportion. A cet égard, la photographie au cerfvolant présente l'intérêt d'une mise en œuvre aisée (quelques séances d'entrainement suffisent à maitriser le vol et la prise de vues). Dans des conditions de vent favorables, quelques minutes suffisent pour positionner l'appareil photo à la verticale de la cible et à la hauteur souhaitée. Seule l'autonomie des batteries de l'appareil photo limitent le temps d'acquisition. Les risques de chute au sol du matériel, sans être nulles, restent limités: en douze ans de pratique, nous n'avons du déplorer qu'une seule chute ayant provoqué la destruction de l'appareil photo. Ce risque est d'autant plus relatif que le matériel exposé reste d'un coût peu élevé. La relative rusticité du matériel dans son ensemble réduit les sources de panne. Le risque existe mais peut être largement limité par un contrôle méthodique avant le vol: charge des batteries de l'appareil photo et de la radiocommande, bon positionnement du servo-moteur qui agit sur le déclencheur, contrôle des paramètres de prise de vue... Plus le système est simple, moins les risques de panne sont élevés. À cet égard, on peut mentionner une variante de la technique encore plus simple pour les prises de vue à faible hauteur, mais qui, en archéologie ne concerne pas la moindre des échelles: celle du chantier de fouille. II s'agit d'utiliser une canne à pêche du commerce (ou n'importe quelle ustensile faisant office de perche) qui permet de positionner l'appareil photo à plusieurs mètres au dessus du chantier (figure 13). Un simple appareil photo doté d'un intervallomètre suffit mais les résultats restent excellents !

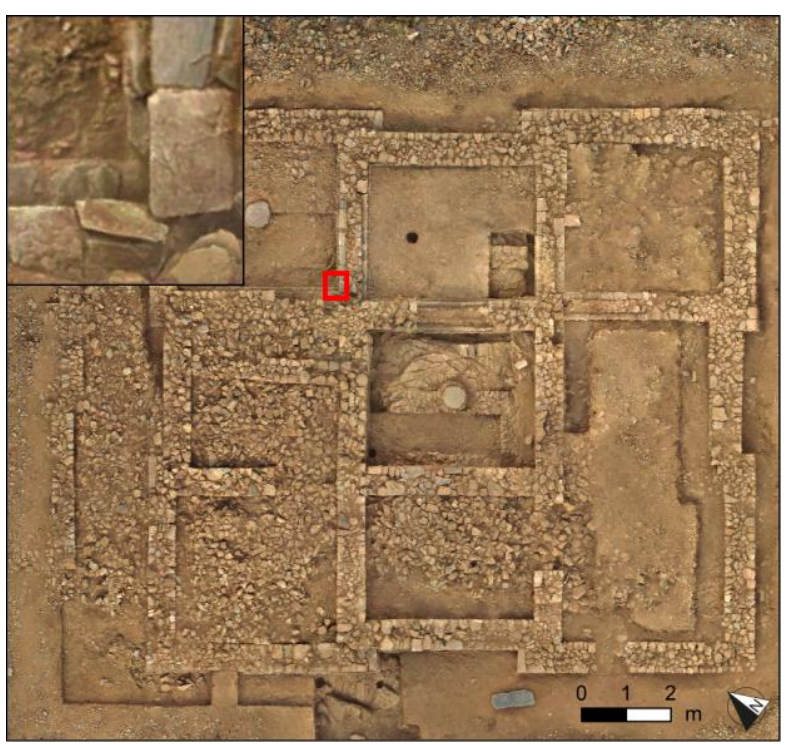

Figure 13 : Ortho-photo obtenu à partir de photos prises à l'aide d'une perche d'une maison d'époque aksumite à Wakarida (Éthiopie) : la résolution permet de distinguer nettement tous les matériaux de la construction.

Dans le cadre d'une mission archéologique à l'étranger, il est souvent difficile d'acheminer le matériel ou de mettre en œuvre des techniques sophistiquées. Le matériel est soumis à rude épreuve (poussière en 
particulier) et les conditions de travail sont souvent rustiques: il est arrivé des cas où la seule source d'approvisionnement en électricité était la batterie du véhicule. Un matériel simple limite donc les contraintes de transport et de mise en œuvre. Un matériel simple présente aussi l'avantage d'un passage en douane facilité. Le transport d'un cerf-volant, à l'inverse des matériels bardés de hautes technologies, n'éveille aucunement la méfiance des douaniers et évite que le matériel ne soit consigné. En effet, même avec les autorisations en bonne et due forme et pour d'obscures raisons, il nous est arrivé à plusieurs reprises que nos GPS ou stations totales ne franchisent pas les portes de l'aéroport; même si le matériel nous fut in fine toujours restitué, il est arrivé que plusieurs semaines s'écoulent avant qu'il ne soit opérationnel sur le terrain.

Enfin, si les pays dans lesquels nous travaillons n'ont pas mis en place de règlementation contraignante visà-vis des techniques légères de prise de vue aérienne, l'obtention d'une autorisation de survol avec un engin motorisé nécessiterait, au mieux, de longues et fastidieuses démarches auprès des autorités. II va sans dire que dans la plupart des cas, compte tenu des usages militaires qui en sont fait, il est inutile d'imaginer obtenir une autorisation pour utiliser un drone. L'usage de ballons captifs ne poserait pas ce genre de problème et les contraintes liées à leur mise en oeuvre sont proches de celles des cerfs-volants. II est cependant souvent compliqué de s'approvisionner en hélium, tout au moins à un prix raisonnable. Pour toutes ces questions matérielles, logistiques ou réglementaires, les cerfs-volants constituent dans la majorité des cas le seul moyen d'acquisition de photographies aériennes à très haute résolution.

\section{Conclusion}

Le cerf-volant est un engin de loisir et prétendre l'utiliser à des fins professionnelles et scientifiques provoque souvent le sourire facétieux de nos interlocuteurs. II serait faux de dire que l'on n'éprouve aucun plaisir à sa mise en œuvre mais cela n'enlève rien à la qualité des clichés obtenus, ni à l'étonnante précision des documents qui en sont issus. Certes, la simplicité de la technique présente des limites : le contrôle du vol n'est pas suffisant pour garantir une localisation et une hauteur parfaitement calibrées. Le poids limité qu'il est possible d'embarquer contraint également à utiliser des appareils photo du commerce qui ne sont pas nécessairement les plus performants pour réaliser un traitement photogrammétrique. Ce dernier fait appel à un logiciel d'emploi aisé dont les résultats n'égalent certainement pas ceux délivrés par les logiciels de spécialistes. Toutefois, l'immense majorité des travaux archéologiques n'ont pas pour cadre des sites dont le prestige, l'intérêt patrimonial ou les impératifs de conservation imposent la mise en œuvre des techniques les plus sophistiquées. A cet égard, les résultats obtenus montrent que leur précision et leur résolution dépassent celles escomptées en regard des besoins courants et des questions posées.

L'emploi d'un logiciel simple et d'un appareil photo du commerce sont cohérents avec la relative rusticité de la technique de prise de vues. L'ensemble ne requiert pas de personnel spécialisé et peut être mis en œuvre au moment opportun par une personne intervenant par ailleurs sur les chantiers archéologiques. II ne présente aucun coût en dehors de l'acquisition initiale du matériel. Le seul appareillage coûteux qu'implique la démarche est le matériel de mesure précis des coordonnées des points de calage (station totale ou DGPS), mais ces matériels sont de toute façon présents sur les chantiers archéologiques. Pour toutes ces raisons, le cerf-volant est parfaitement adapté aux missions de terrain à l'étranger, tout au moins celles ayant pour cadre les milieux arides.

Dans le cadre métropolitain, d'ailleurs contraint par la règlementation, nombreux sont les sites aux environnements dégagés. Comme à l'étranger, la plupart des problématiques ne requièrent pas des précisions et des résolutions qui dépassent celles obtenues à partir d'un cerf-volant. Pour autant que son emploi ne soit pas associé trop hâtivement au loisir mais à un outil simple, efficace et peu couteux, il pourrait parfaitement faire partie de la panoplie des outils de relevés employés au quotidien sur les chantiers.

\section{Références}

Barge O., Régagnon E., Penser le plan archéologique comme un système d'information, L'exemple du site médiéval de Qalhat (Oman), Revue internationale de Géomatique, VOL 22/3, 343-365.

Cauvin C., Escobar F., Serradj A., 2007. Cartographie thématique 1 : une nouvelle démarche, Paris, Lavoisier, pp 208 (a), pp. 207 (b).

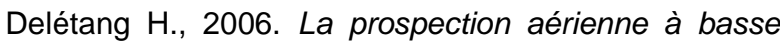
altitude, La prospection, Errance.

Favory F., 1998. La Télédétection satellitaire appliquée à l'archéologie. Les Nouvelles de l'Archéologie, 74:551.

Giraud J., Charbonnier J., Gernez G., Lemee M., Righetti S., 2012. Occupation ancienne dans la région d'Ấdam (Sultanat d'Oman) du Néolithique à la période pré-islamique, Chroniques Yéménites 17.

Jung C., 2006. La photo- et la carto-interprétation, La prospection, Errance.

Nègre S., 1999. Vu du ciel : la photographie aérienne par cerf-volant, Labruguière, Société d'études et de recherches archéologiques et historiques de Labruguière.

Rodier X. (dir.), Barge O., Saligny L., Nuninger L., Bertoncello F., 2011. Information spatiale et archéologie, "Archéologiques", Errance. 